\title{
The Application of Continuous Spatial Scaling Model of NDVI in Validation and Its Sensibility Analysis to Various Image Characteristics
}

\author{
Haijun Luan ${ }^{1,2}$, Qingjiu Tian ${ }^{2}$, Xinxin Zhang ${ }^{1}$, Qin $\mathrm{Nie}^{1}$, Hui $\mathrm{Li}^{1}$ and Huoping $\mathrm{Pan}^{1}$ \\ 1. College of Computer and Information Engineering, Xiamen University of Technology, Xiamen 361024, China \\ 2. International Institute for Earth System Science, Nanjing University, Nanjing 210023, China
}

\begin{abstract}
Based on the fractal theory, this study establishes a Continuous Spatial Scaling Model (CSSM) of the Normalized Difference Vegetation Index (NDVI) to address issues arising with spatial up-scaling in quantitative remote sensing. This model is able to quantitatively describe transformation relationships of the NDVI on continuous scales. Then the following experiments are accomplished: (1) the validation of ETM+ NDVI imagery is implemented based on the GEOEYE-1 image and its NDVI CSSM, and the following conclusion is obtained: because of bad stripes in the ETM+ image and the limited effect of destriping, the ETM+ NDVI image had a rather large error, and the error for the entire experimental imagery is about $25 \%$, so the ETM+ NDVI product is not suitable for direct practical application; (2) Shatian Byland (Beihai City, China) is taken as the experimental area, and four images (two ETM+ images with wider and smaller coverage, respectively, a GEOEYE-1 image, and an HJ-1B CCD1 image) are studied. The most suitable scale levels are computed and compared for the four images, and a better understanding is obtained of the impact of various image characteristics (area of coverage, spatial resolution, and imaging quality) on determining the scale level for the NDVI CSSM.
\end{abstract}

Key words: Normalized Difference Vegetation Index (NDVI), scale transformation, similarity fractal dimension, validation, image characteristics.

\section{Introduction}

Spatial scale transformation is one of the most important basic issues arising in quantitative remote sensing [1]. In particular, spatial up-scaling has attracted much attention, since it can provide an effective way to solve a number of difficult problems, such as validation of quantitative remote sensing products [2-4]. There is no doubt that land surface reflectance presented scale effects $[5,6]$. As the nonlinear combination of reflectance on red and near-infrared bands, the Normalized Difference Vegetation Index (NDVI) has more obvious and complex scale effects. Some studied it with statistic methods [7-11], and others utilized analytical methods such as Taylor Series Expansion (TSE) [12, 13].

Corresponding author: Haijun Luan, Ph.D., main research field: scale effects research of quantitative remote sensing.
However, as an important retrieval, the use of up-scaling for NDVI is confronted by these two issues in its implementation: (1) although the statistical methods were applied widely which could solve concrete problems well, the transformation formulas required lots of sample data and did not have clear physical meanings which were not applicable for different zones or sensors; (2) although the analytical methods had clear mathematical meanings, their kinds were limited and their precision was restricted because of some simplification and hypothesis conditions.

Based on the fractal theory, this study establishes a Continuous Spatial Scaling Model (CSSM) of the NDVI to address the above issues. This model is able to quantitatively describe the transformation relationships of the NDVI on continuous scales. Furthermore, the impact of different image characteristics (coverage 
area, spatial resolution, and imaging quality) on determining the most reasonable scale level for establishing the NDVI. CSSM is subjected to a thorough analysis.

\section{Materials and Methods}

\subsection{Materials}

The experimental area was located in the Shatian Byland of Eastern Hepu County, Beihai City in the Guangxi Zhuang Autonomous Region of China, which is located at $109^{\circ} 42^{\prime} 3^{\prime \prime} \mathrm{E}$ to $109^{\circ} 45^{\prime} 36^{\prime \prime} \mathrm{E}$ and $21^{\circ} 29^{\prime} 0^{\prime \prime}$ to $21^{\circ} 33^{\prime} 25^{\prime \prime}$. During the time at which the experimental images were acquired, from early to late October, the coverage in this zone comprised mainly eucalyptus, natural forest, mangrove, cassava, paddy fields, man-made constructions and water. This zone was selected for the experiment precisely because it is for such situations with different kinds of land object and with high spatial heterogeneity scaling of the NDVI using a fractal method which has potential advantages.

There were three original images: (a) a $2 \mathrm{~m}$ GEOEYE-1 image acquired on October 16, 2009, of 4,112 × 3,036 pixels; (b) a $30 \mathrm{~m}$ HJ-1B CCD1 image acquired on October 7, 2009; (c) a $30 \mathrm{~m}$ Landsat7 ETM+ image acquired on October 23, 2009. Preprocessing of these images was implemented as follows:

Atmospheric correction of the GEOEYE-1 image was done based on the ENVI FLAASH module, and its land surface radiance (Fig. 1) was obtained according to the transformation formula land surface radiance $=$ land surface incident radiance $\times$ land surface hemispherical-directional reflectance [14] using 6S software.

The land surface radiance of the HJ-1B CCD1 image was obtained using the same method, and geometrical correction of the image was done with reference to the GEOEYE-1 image. Then, after preprocessing, the zone of the HJ-1B CCD1 image corresponding to the GEOEYE-1 image was taken as the experimental image (Fig. 2).

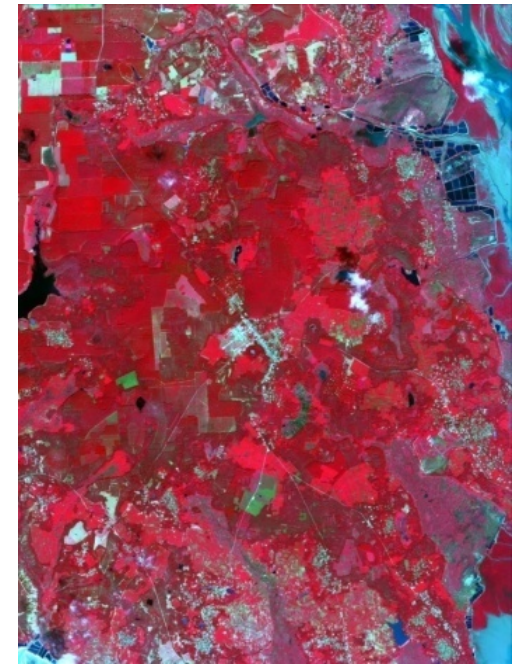

Fig. 1 The GEOEYE-1 false-color composite image (R: 4, G: 3, B: 2) of the experimental area after preprocessing.

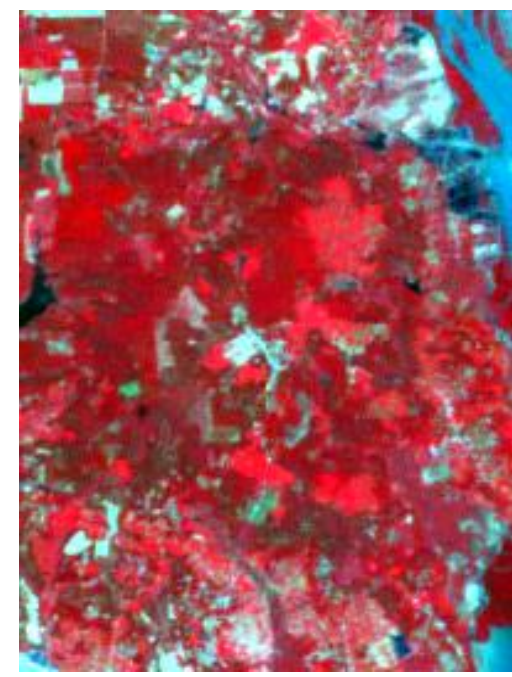

Fig. 2 The HJ-1B CCD1 false-color composite image (R: 4, G: 3, B: 2) of the experimental area after preprocessing.

First, the ETM+ image was destriped, and then its land surface radiance was obtained by the same method as for the other two images. Finally, a $400 \times 300$ pixel zone and a $272 \times 200$ pixel zone (the same area as the GEOEYE-1 image) were taken as experimental images, which will henceforth be referred to respectively as the ETM+ image with wider coverage and the ETM+ image with smaller coverage (Fig. 3).

The four post-processed images represent examples of images with different characteristics, namely, area of coverage (the ETM+ image with wider coverage versus the ETM+ image with smaller coverage), spatial resolution (the GEOEYE-1 image versus the ETM+ 


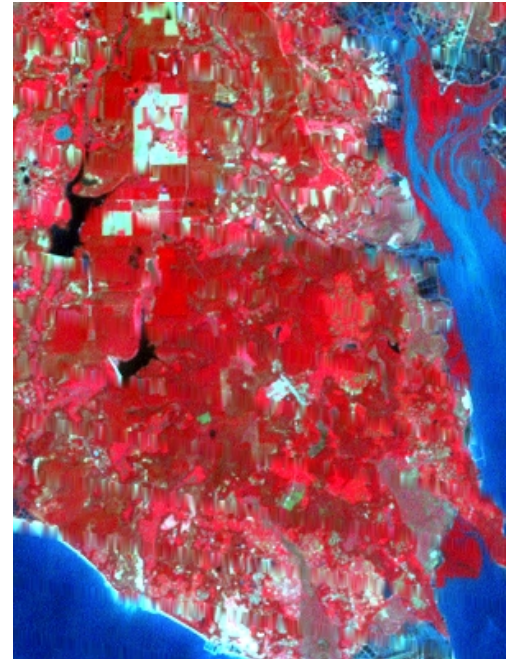

(a) The ETM+ image with wider coverage $(400 \times 300$ pixels $)$.

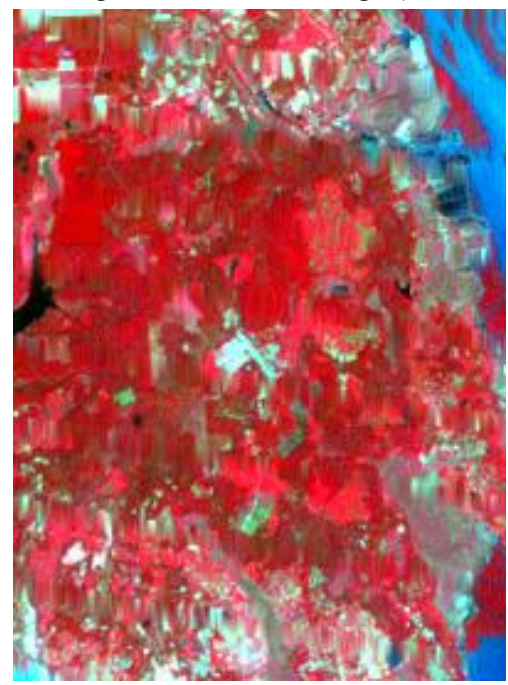

(b) The ETM+ image with smaller coverage $(272 \times 200$ pixels $)$.

Fig. 3 The ETM+ false-color composite images (R: 4, G: 3, B: 2) of the experimental area after preprocessing.

image with smaller coverage), and imaging quality (the HJ-1 CCD1 image versus the ETM+ image with smaller coverage). They can therefore be used to investigate the impact of these characteristics on determining the most reasonable scale level of the NDVI CSSM.

\subsection{Methodology}

The similarity fractal dimension was defined by

$$
D=\log _{\frac{1}{s}} u=\frac{\lg u}{\lg \frac{1}{S}}
$$

and, using this, the scale and NDVI parameters were transformed. According to Fig. 4, the CSSM of the NDVI was then established as follows. First, the land surface radiances (e.g., $r_{-} 11, r_{-} 12, r_{-} 21$ and $\left.r_{-} 22\right)$ on the scale of the basic image (e.g., the $2 \mathrm{~m}$ scale of the GEOEYE-1 image) were summed by area to obtain the land surface radiance rad on larger scales, and the mean of NDVI $(N D V I)$ on these larger scales was computed. Second, taking the basic image scale (e.g., the $2 \mathrm{~m}$ scale of the GEOEYE-1 image) as the base, the scale factor (scale) of the similarity fractal dimension was defined as the larger scale (scale_up) divided by the basic image scale (e.g., scale_2). Third, by computing the $\log _{2}$ arrays of $1 /$ scale and $N D V I$, a linear regression was performed based on the arrays:

$$
\log _{2} N D V I=d \log _{2} \frac{1}{\text { scale }}+b
$$

where $d$ is the slope of the fitting line and the constant $b$ is its intercept. The NDVI CSSM was then established as

$$
N D V I=2^{b}\left(\frac{1}{\text { scale }}\right)^{d}
$$

and its similarity fractal dimension D was calculated as

$$
D=2-d
$$

Because the land surface radiance satisfied the up-scaling rule of summing according to area [17], the NDVI model with land surface radiance was selected to

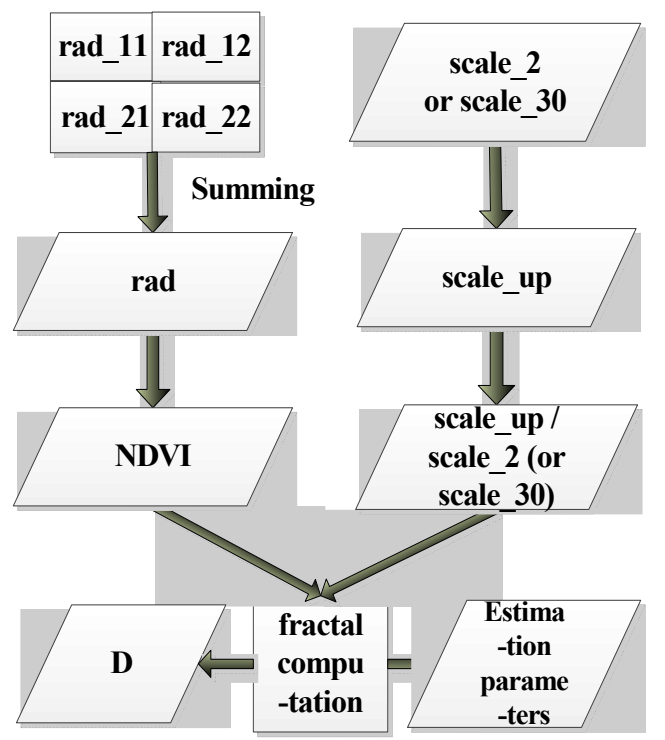

Fig. 4 Flowchart of the model for continuous scaling of the NDVI by a fractal method $[15,16]$. 
establish a physically meaningful CSSM. A statistical estimation procedure was then applied to this model, with four relatively independent and related statistical parameters $(r, p, r l o, r u p)$ being chosen. Here, $r$ is the correlation coefficient between the fitting line and the true curve in Eq. (2), valid for small $p$-values ( $p<0.05$ ), while rlo and rup are respectively the lower and upper limits of the confidence interval around $r=0.95$, according to which the possibility is less than 0.05 that the true $r$ is less than $r l o$.

Furthermore, on a different large scale, the difference between NDVI from Eq. (3) and the near-true value can be computed as [15]

$$
\text { Diff }=N D V I_{1}-N D V I_{2}
$$

where $N D V I_{1}$ is the mean NDVI on the larger scale from Eq. (3) and $N D V I_{2}$ is the near-true mean NDVI on the same scale (e.g., the mean of the NDVI image computed from the true land surface radiance image). Here, Diff represents the error in $N D V I_{1}$. Thus, the ratio of Diff to $N D V I_{2}$,

$$
\text { Error }=\text { Diff } / N D V I_{2}
$$

represents the ratio of the error in $N D V I_{1}$ to $N D V I_{2}$. On this basis, Max_of_abs (Error) can be defined as the largest absolute value of Error, which is the largest error in validating NDVI using the CSSM, and can therefore be chosen as an index of the applicability of the CSSM.

Taking the four statistical indices ( $r, p$, rlo and rup) together with the index Max_of_abs (Error), the CSSM can be comprehensively estimated on the basis of statistics and applicability. These indices are mutually dependent and form a five-index estimation system for the NDVI CSSM. According to the flowchart shown in Fig. 4, different large scales can be obtained from the basic image scale (e.g., the $2 \mathrm{~m}$ of the GEOEYE-1 image), with the largest of these scales corresponding to the final scale level. For a basic image (e.g., GEOEYE-1), different final scale levels can be set, and different NDVI CSSMs can be established for each of these. From these models, the most appropriate NDVI CSSM can be chosen once the parameter values for the estimation system have been set.

In this study, the appropriate scale levels for the experimental images were defined as follows. First, the NDVI CSSM of an image was established at each level. Second, the NDVI CSSMs at each level were analyzed based on the standard criterion $(r \geq 0.8, p<0.05$ and $r l o$ $\geq r \leq$ rup), and the levels matching the criterion were selected. Third, with the aim of determining the NDVI CSSM with the widest range of scales, based on the other criterion Max_of_abs(Error) $\leq 0.05$, the most appropriate scale level could be defined from the available scale levels computed above.

To quantitatively analyze the reason for the NDVI scale effect, a spatial heterogeneity index $(S H I)$ was defined to estimate the spatial heterogeneity of the images [18]. The $S H I$ of an image pixel $f(i, j)$ is defined as the sum of the absolute differences between a pixel and its eight neighbors:

$$
S H I_{i j}=\sum_{a=-1}^{1} \sum_{b=-1}^{1}|f(i, j)-f(i+a, j+b)|
$$

Thus, Mean SHI for an image of size $m \times n$ pixels can be computed as

$$
\text { Mean } S H I=\frac{1}{m \cdot n} \sum_{i=1}^{m-1} \sum_{j=1}^{n-1} S H I_{i j}
$$

In general, the greater the value of Mean SHI for an NDVI image is, the more obvious the scale effect of the image is.

\section{Results and Discussion}

\subsection{Experiments and Results}

The following experimental results were obtained.

(a) Validation of ETM+ NDVI imagery based on the GEOEYE-1 image and its NDVI CSSM

In the experiment, the NDVI CSSM of the GEOEYE-1 image (Fig. 1) was established on the basis of fractal theory and a five-index estimation system $(r$ $\geq 0.8, p<0.05, r l o \geq r \leq$ rup and Max_of_abs(Error) $\leq$ $0.05)$, and, by integrating the spectral normalization, the ETM+ NDVI image (Fig. 3b) was validated. From 
each image, 22 sample images of same areas were selected, which had four kinds of ranges (150 pixel $\times$ 150 pixel, 600 pixel $\times 600$ pixel, 1,500 pixel $\times 1,500$ pixel and 4,112 pixel $\times 3,036$ pixel for GEOEYE-1 image, which were respectively corresponded to 10 pixel $\times 10$ pixel, 40 pixel $\times 40$ pixel, 100 pixel $\times 100$ pixel and 272 pixel $\times 200$ pixel of ETM + image) and contained different kinds of ground objects described in "2.1 Materials". Then, on a $30 \mathrm{~m}$ scale, the NDVI values from the model and the ETM+ sample images were computed and compared, as shown in Fig. 5a. Fig. $5 \mathrm{~b}$ shows the NDVI errors for the ETM + sample images computed from Eq. (6) and compared with the upper and lower limits of acceptable error $( \pm 0.05)$, which were defined according to the estimation criterion Max_of_abs(Error) $\leq 0.05$. The experimental results were analyzed, with the following conclusion: because of the influence of the bad stripes in the ETM+ image and the limited effect of destriping, the ETM+ NDVI image had a rather large error, and the error for the entire experimental imagery was about $25 \%$, so the ETM+ NDVI product was not suitable for direct practical application. Meanwhile, the potential value of NDVI CSSM for validation was demonstrated.

For the ETM+ images with wider and smaller coverage, the NDVI CSSMs were respectively established at the most suitable scale levels (Levels 267 and 181) (Figs. 6 and 9), their Errors were computed (Figs. 7 and 10), and the SHIs of the two NDVI images were computed (Figs. 8 and 11).

(b) Comparison of NDVI CSSMs based on the ETM+ images with wider and smaller coverage

In Figs. 7 and 10, the absolute values of Errors are generally non-zero: for the ETM+ image with wider coverage, the minimum absolute value is $3.6308 \times 10^{-5}$ (at $u p$-scale $=5,370 \mathrm{~m}$ ) and the maximum is 0.0497 (at up-scale $=30 \mathrm{~m})$, and for the ETM+ image with smaller coverage, the minimum is $1.3217 \times 10^{-5}$ (at up-scale $=630 \mathrm{~m}$ ) and the maximum is 0.0237 (at up-scale $=3,000 \mathrm{~m}$ ). For the most suitable NDVI CSSMs, the maximum absolute values of Errors are

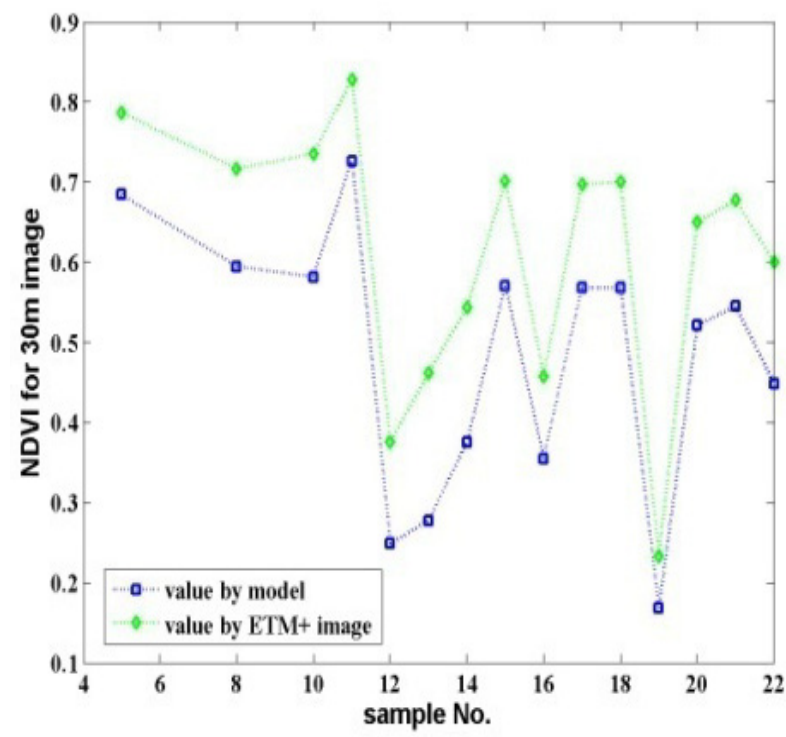

(a) Comparison of NDVI values according to the model and $\mathrm{ETM}+$ sample images.

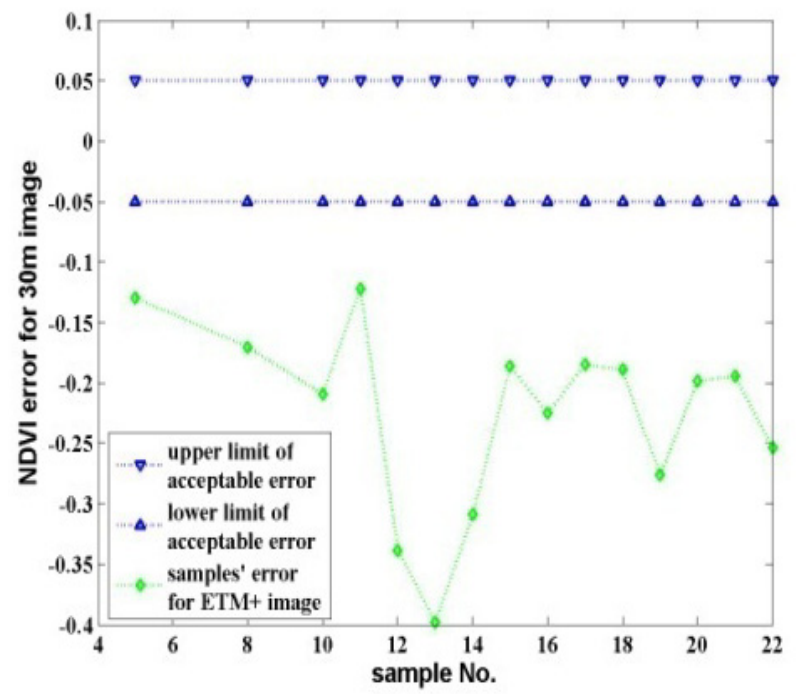

(b) NDVI errors for the ETM+ sample images.

Fig. 5 Validation of ETM+ NDVI.

less than 0.05 , which is consistent with the criterion Max_of_abs(Error) $\leq 0.05$, and therefore the CSSMs can be applied for validation of the NDVI. In Figs. 8 and 11, Mean SHI at different up-scales would be the same if the ETM+ NDVI images were spatially uniform and not heterogeneous. In both of these figures, however, Mean SHI varies clearly on different scales, indicating that the ETM+ NDVI images are of high spatial heterogeneity, which is the reason for the NDVI scale effect and the Errors generated. 

Sensibility Analysis to Various Image Characteristics

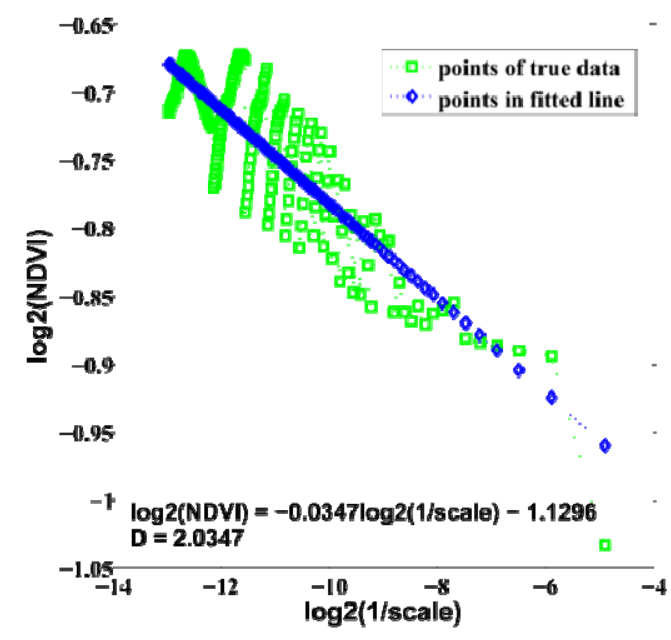

Fig. 6 Establishing the NDVI CSSM at the most suitable scale level $($ Level $=267)$ for the ETM+ image with wider coverage.

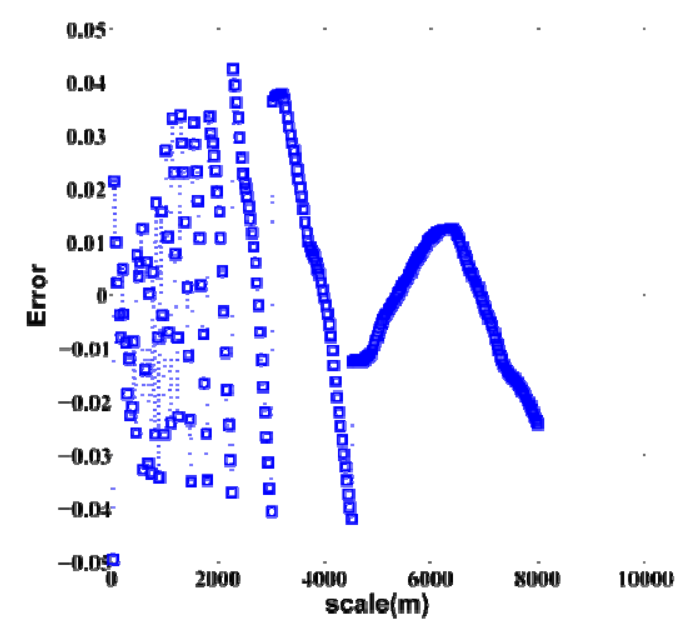

Fig. 7 Difference between the NDVI results from the two methods $($ Level $=267)$ for the ETM+ image with wider coverage.

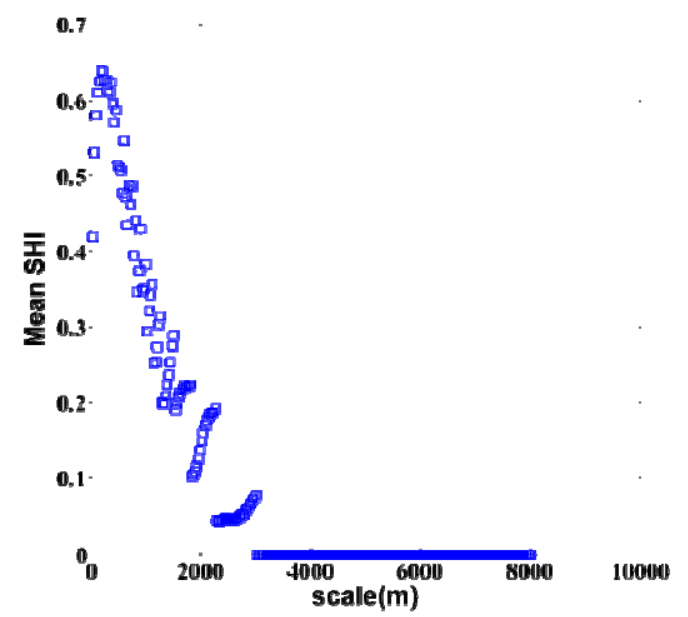

Fig. 8 The scale versus SHI curve of the image (Level = 267) for the ETM+ image with wider coverage.

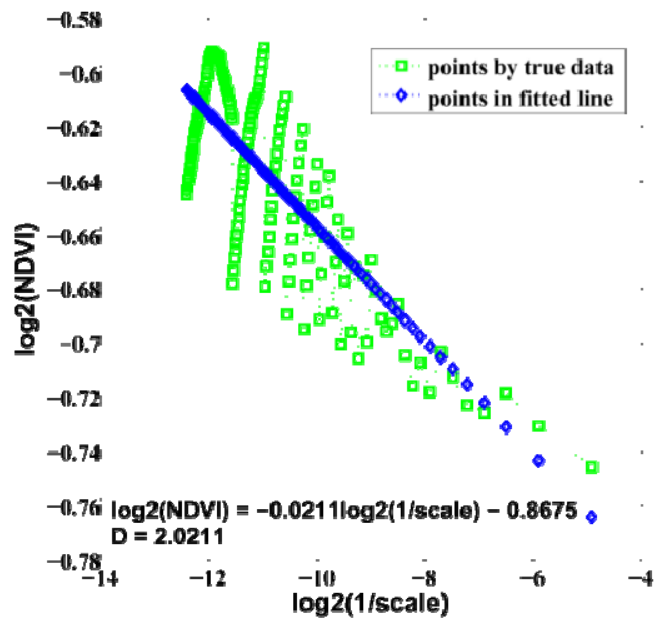

Fig. 9 Establishing the NDVI CSSM at the most suitable scale. level $($ Level $=181)$ for the ETM+ image with smaller coverage.

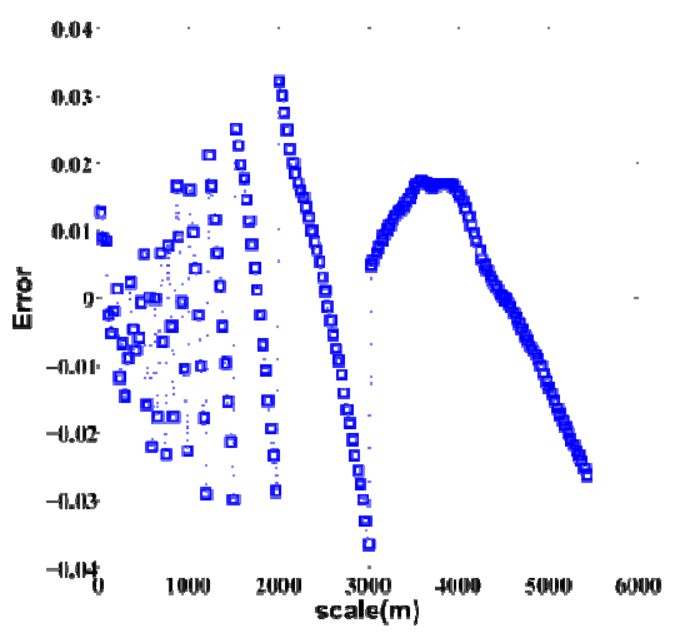

Fig. 10 Difference between the NDVI results from the two methods $($ Level $=181)$ for the ETM+ image with smaller coverage.

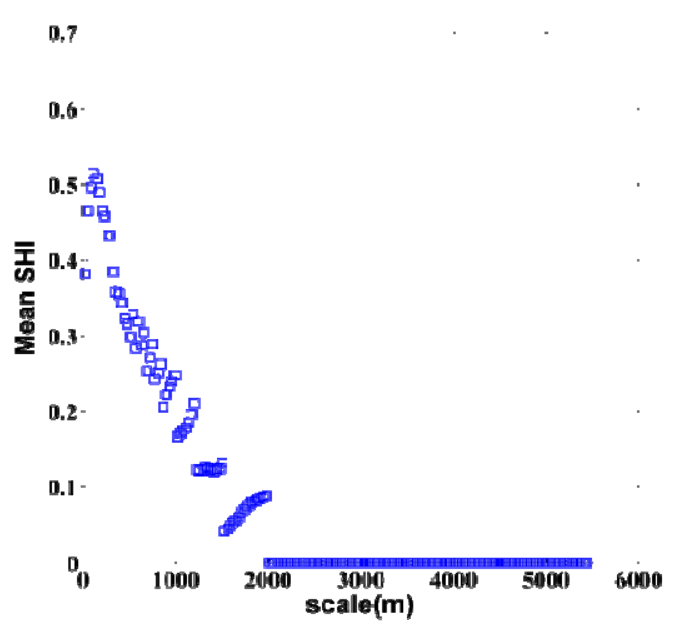

Fig. 11 The scale versus SHI curve of the image (Level = 181) for the ETM+ image with smaller coverage. 
Table 1 Comparison of the NDVI CSSMs based on the ETM+ Images with wider and smaller coverage.

\begin{tabular}{llllll}
\hline Image & Mean SHI & Most suitable model & $\begin{array}{l}\text { Fractal } \\
\text { dimension }\end{array}$ & Scale range & Error range \\
\hline $\begin{array}{l}\text { ETM+ image } \\
\text { with wider } \\
\text { coverage }\end{array}$ & 0.4200 & $\log _{2}$ NDVI $=-0.0347 \log _{2} \frac{1}{\text { scale }}-1.1296$ & 2.0347 & $30 \mathrm{~m}$ to $8,010 \mathrm{~m}$ & $\begin{array}{l}3.6308 \times 10^{-5} \text { to } \\
0.0497\end{array}$ \\
$\begin{array}{l}\text { ETM+ image } \\
\text { with smaller } \\
\text { coverage }\end{array}$ & 0.3823 & $\log _{2}$ NDVI $=-0.0211 \log _{2} \frac{1}{\text { scale }}-0.8675$ & 2.0211 & $30 \mathrm{~m}$ to $5,430 \mathrm{~m}$ & $\begin{array}{l}1.3217 \times 10^{-5} \\
\text { to } 0.0237\end{array}$ \\
\hline
\end{tabular}

On this basis, and taking the mean SHI, the most suitable model, the fractal dimension, the scale range, and the error range as indices, the NDVI CSSMs of the ETM+ images with wider and smaller coverage are compared in Table 1.

It can be concluded from Table 1 that, for images from the same sensor with similar land cover type, the wider the area of coverage is and the greater the spatial heterogeneity of the image is; the higher the scale level of the model is and the wider its scale range is.

(c) Comparison of NDVI CSSMs based on the GEOEYE-1 image and ETM+ image with smaller coverage

For the GEOEYE-1 image and the ETM+ image with smaller coverage, the NDVI CSSMs were respectively established at the most suitable scale levels (Levels 495 and 181) (Figs. 9 and 12). Their Errors were computed (Figs. 10 and 13), and the SHIs of the two NDVI images were computed (Figs. 11 and 14).

In Figs. 13 and 10, the absolute values of Errors are generally non-zero: for the GEOEYE-1 image, the minimum absolute value is $1.0697 \times 10^{-5}$ (at up-scale $=$ $448 \mathrm{~m}$ ) and the maximum is 0.0127 (at up-scale $=868$ $\mathrm{m})$, and for the ETM+ image with smaller coverage, the minimum is $1.3217 \times 10^{-5}$ (at up-scale $=630 \mathrm{~m}$ ) and the maximum is 0.0237 (at up-scale $=3,000 \mathrm{~m}$ ). For the most suitable NDVI CSSMs, the maximum absolute values of Errors are less than 0.05, which is consistent with the criterion Max_of_abs(Error) $\leq 0.05)$, and therefore the CSSMs can be applied to validation of the NDVI. In Figs. 11 and 14, the Mean SHI at different up-scales would be the same if the GEOEYE-1 and ETM+ NDVI images were spatially uniform and not heterogeneous. In both of these figures, however, Mean
SHI varies clearly on different scales, indicating that the two NDVI images are of high spatial heterogeneity, which is the reason for the NDVI scale effect and the Errors generated.

On this basis, taking the mean $S H I$, the most suitable model, the fractal dimension, the scale range, and the

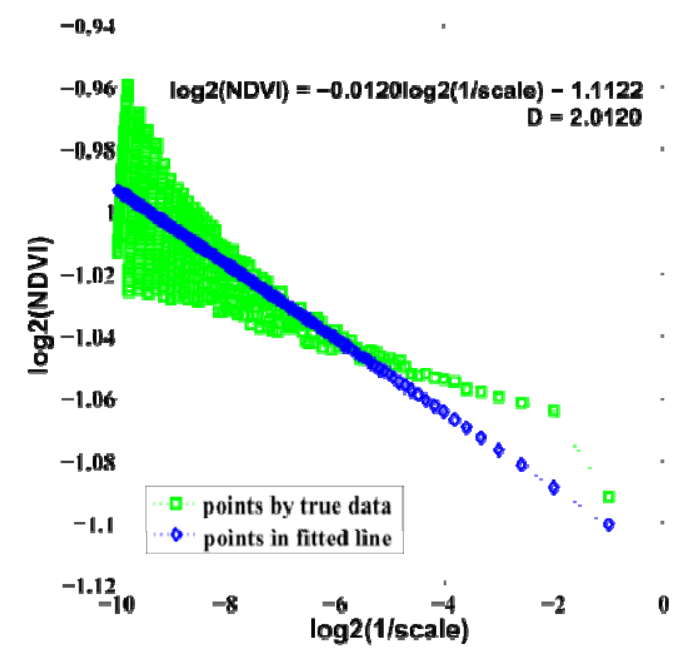

Fig. 12 Establishing the NDVI CSSM at the most suitable scale level $($ Level $=495)$ for the GEOEYE-1 image.

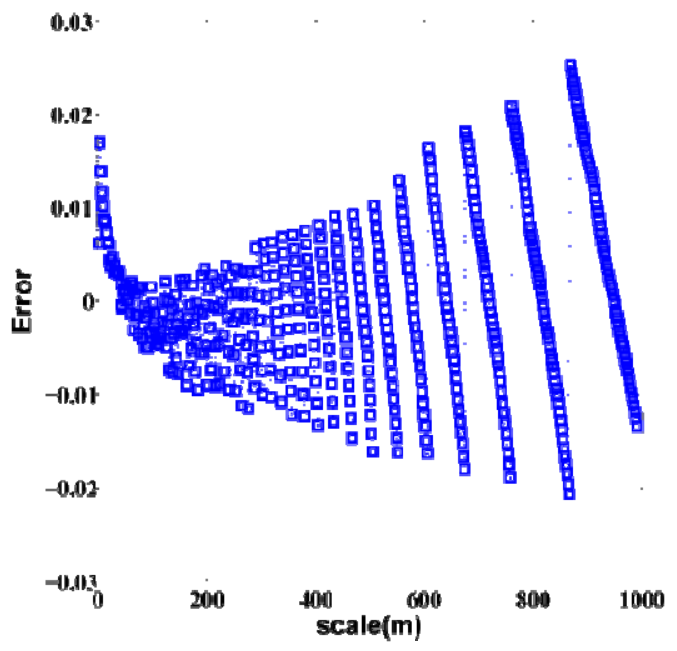

Fig. 13 Difference between the NDVI results from the two methods (Level $=495)$ for the GEOEYE-1 image. 


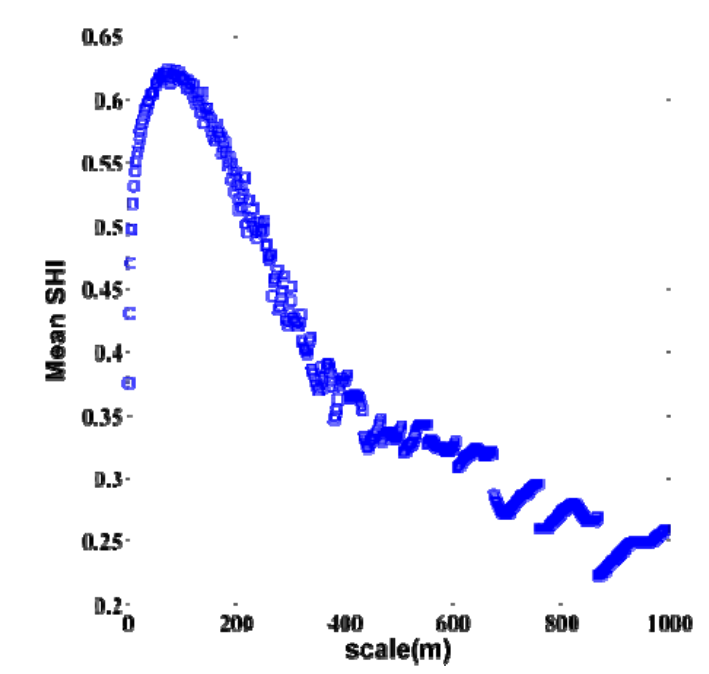

Fig. 14 The scale versus SHI curve of the image (Level $=$ 495) for the GEOEYE-1 image.

error range as indices, the NDVI CSSMs of the GEOEYE-1 image and the ETM+ image with smaller coverage are compared in Table 2.

It can be concluded from Table 2 that, for images from different sensors of the same area of coverage and the same land cover type, the higher the spatial resolution is and the lower the spatial heterogeneity of the image is; the finer the scale of the model is but the smaller its scale range is, starting from a smaller scale corresponding to the source image's resolution and containing scales corresponding to integer multiples of the source image resolution. Conversely, the lower the spatial resolution is and the higher the spatial heterogeneity of the image is; the coarser the scale of the model is but the wider its scale range is, starting from a larger scale corresponding to the source image resolution and containing scales corresponding to integer multiples of the source image resolution.

Comparison of NDVI CSSMs based on the HJ-1 CCD1 image and ETM+ image with smaller coverage
For the HJ-1 CCD1 image and the ETM+ image with smaller coverage, the NDVI CSSMs were respectively established at the most suitable scale levels (Levels 24 and 181) (Figs. 9 and 15). Their Errors were computed (Figs. 10 and 16), and the SHIs of the two NDVI images were computed (Figs. 11 and 17).

In Figs. 10 and 16, the absolute values of Errors are generally non-zero: for the HJ-1 CCD1 image, the minimum absolute value is $2.3396 \times 10^{-4}$ (at up-scale $=$ $180 \mathrm{~m}$ ) and the maximum is 0.0068 (at up-scale $=600$ $\mathrm{m})$, and for the ETM+ image with smaller coverage, the minimum is $1.3217 \times 10^{-5}$ (at up-scale $=630 \mathrm{~m}$ ) and the maximum is 0.0237 (at up-scale $=3,000 \mathrm{~m}$ ). For the most suitable NDVI CSSMs, the maximum absolute values of Errors are less than 0.05, which is consistent with the criterion Max_of_abs(Error) $\leq 0.05$, and therefore the CSSMs can be applied to validation of the NDVI. In Figs. 11 and 17, the Mean SHI at different up-scales would be the same if the HJ-1 CCD1 and ETM+ NDVI images were spatially uniform and not heterogeneous. In both of these figures, however, Mean $S H I$ varies clearly on different scales, indicating that the two NDVI images are of high spatial heterogeneity, which is the reason for the NDVI scale effect and the Errors generated.

On this basis, taking the mean $S H I$, the most suitable model, the fractal dimension, the scale range, and the error range as indices, the NDVI CSSMs of the HJ-1 CCD1 image and the ETM+ image with smaller coverage are compared in Table 3.

It can be concluded from Table 3 that, for images from different sensors of the same area of coverage and land cover type, the better the imaging quality is and the better the capability to describe complex structure

Table 2 Comparison of NDVI CSSMs based on the GEOEYE-1 image and the ETM+ image with smaller coverage.

\begin{tabular}{llllll}
\hline Image & Mean SHI & Most suitable model & $\begin{array}{l}\text { Fractal } \\
\text { dimension }\end{array}$ & Scale range & Error range \\
\hline $\begin{array}{l}\text { GEOEYE-1 } \\
\text { image }\end{array}$ & 0.3746 & $\log _{2}$ NDVI $=-0.0120 \log _{2} \frac{1}{\text { scale }}-1.1122$ & 2.0120 & $2 \mathrm{~m}$ to $990 \mathrm{~m}$ & $\begin{array}{l}1.0697 \times 10^{-5} \\
\text { to } 0.0127\end{array}$ \\
$\begin{array}{l}\text { ETM+ image } \\
\text { with smaller } \\
\text { coverage }\end{array}$ & 0.3823 & $\log _{2}$ NDVI $=-0.0211 \log _{2} \frac{1}{\text { scale }}-0.86752 .0211$ & $30 \mathrm{~m}$ to $5,430 \mathrm{~m}$ & $\begin{array}{l}1.3217 \times 10^{-5} \\
\text { to } 0.0237\end{array}$ \\
\hline
\end{tabular}




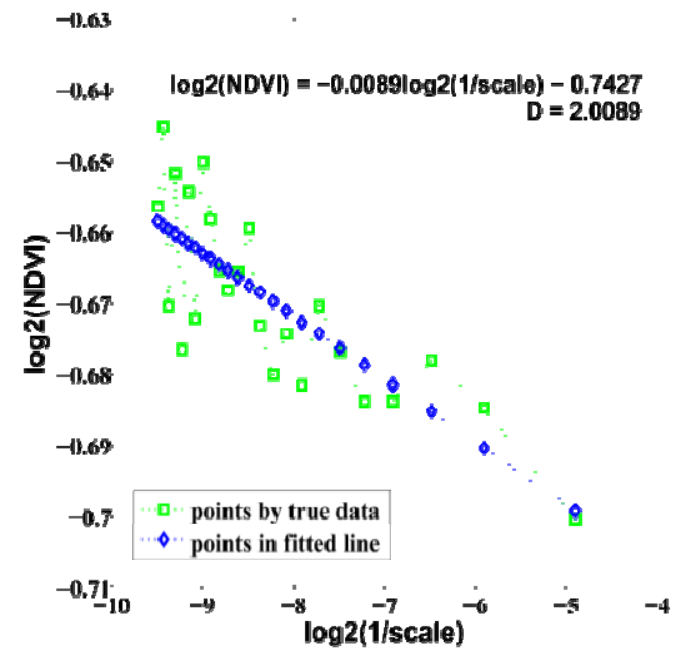

Fig. 15 Establishing the NDVI CSSM at the most suitable scale level $($ Level $=24)$ for the HJ-1 CCD1 image.

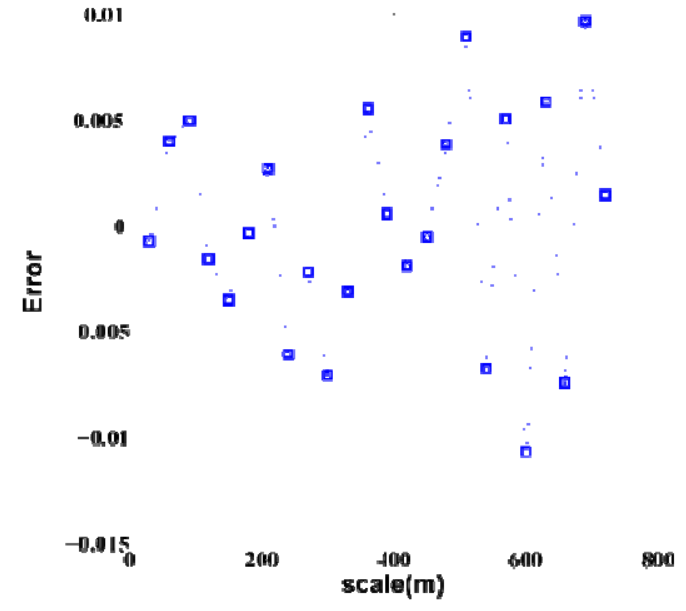

Fig. 16 Difference between the NDVI results from the two methods (Level = 24) for the HJ-1 CCD1 image.

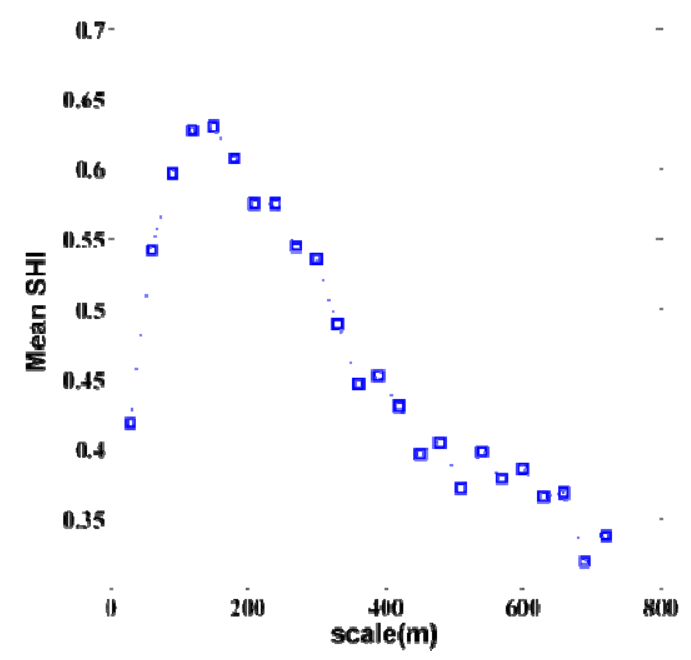

Fig. 17 The scale versus SHI curve of the image (Level = 24) for the HJ-1 CCD1 image. and the spatial heterogeneity of the coverage is; the higher the scale level is and the wider the scale range of the model is.

\subsection{Discussion}

On one hand, from formula 3, the NDVI values by the model are the entire statistical feature of images on each scale, and the CSSM describes the relation between mean NDVIs of images on each scale, not of corresponding pixels. Therefore, it is a whole-image validation method based on NDVI CSSM. Although it is reasonable and reliable [17], which is still anticipated to establish a more precise CSSM describing the relation between corresponding pixels of images on each scale which would implement more accurate validation.

On the other hand, the three sensors have different imaging parameters (e. g. spectral one, temporal one and geometric one), and it would bring some uncertainty into the comparison of NDVI CSSMs for the four experimental images. Consequently, in the next work, the normalization of imaging parameters will be concerned about to capture precise results.

\section{Conclusions}

Based on the fractal theory, this study has established an NDVI CSSM to quantitatively describe the transformation relationships of NDVI on continuous scales, which could be applied into the NDVI's validation. For land surface parameters with summing rules by area, such as land surface radiance and soil moisture, the CSSM methodology (shown in Fig. 4) is readily available from the literature. Furthermore, taking the Shatian Byland as an experimental area and comprehensively utilizing four images (two ETM+ images with wider and smaller coverage, respectively, a GEOEYE-1 image, and an HJ-1B CCD1 image), the impacts of various image characteristics (coverage area, spatial resolution, and imaging quality) on determining the most suitable scale level of NDVI CSSM have been analyzed. The results obtained have led to a better 
Table 3 Comparison of NDVI CSSMs based on the HJ-1 CCD1 image and the ETM+ image with smaller coverage.

\begin{tabular}{llllll}
\hline Image & Mean SHI & Most suitable model & $\begin{array}{l}\text { Fractal } \\
\text { dimension }\end{array}$ & Scale range & Error range \\
\hline $\begin{array}{l}\text { HJ-1 CCD1 } \\
\text { image }\end{array}$ & 0.4186 & $\log _{2}$ NDVI $=-0.0089 \log _{2} \frac{1}{\text { scale }}-0.7427$ & 2.0089 & $30 \mathrm{~m}$ to $720 \mathrm{~m}$ & $\begin{array}{l}2.3396 \times 10^{-4} \\
\text { to } 0.0068\end{array}$ \\
$\begin{array}{l}\text { ETM+ image } \\
\text { with smaller } \\
\text { coverage }\end{array}$ & 0.3823 & $\log _{2}$ NDVI $=-0.0211 \log _{2} \frac{1}{\text { scale }}-0.8675$ & 2.0211 & $30 \mathrm{~m}$ to $5,430 \mathrm{~m}$ & $\begin{array}{l}1.3217 \times 10^{-5} \\
\text { to } 0.0237\end{array}$ \\
\hline
\end{tabular}

understanding of the characteristics of the NDVI CSSM

Furthermore, according to the theory system of fractal geometry [19], fractal measurement and computation is only part of it; the generated mechanism or dynamic process of mathematical fractal and statistical one is another significant part of it. For example, Iterated Function System (IFS) is utilized to reveal the dynamic process of fractal phenomena (e. g. fractal downscaling of soil moisture) [20, 21]. While these researches are preliminary, there are many important problems to be addressed. It will be the research focus in the future work.

\section{Acknowledgment}

This work was supported by the National Natural Science Foundation of China (Nos. 41601350 and 41401475), the Natural Science Foundation of Fujian Province, China (No. 2015J01175), Cooperation with Universities Project of Fujian Bureau of Surveying, Mapping and Geoinformation (No. 2015JX04), and Education and Scientific Research Project (Type A) for Young and Middle-aged Teachers of Fujian Province (No. JA14231).

\section{References}

[1] Li, X., and Wang, Y. 2013. "Prospects on Future Developments of Quantitative Remote Sensing." Acta Geographica Sinica 68 (9): 1163-1169. (in Chinese).

[2] Morisette, J. T., Privette, J. L., and Justice, C. O. 2002. "A Framework for the Validation of MODIS Land Products." Remote Sensing of Environment 83 (1): 77-96.

[3] Liang, S. 2009. Quantitative Remote Sensing. Translated by Fan, W. J. Beijing: Science Press, 180-183. (in Chinese).

[4] Wu, X., Wen, J., Xiao, Q., Li, X., Liu, Q., Tang, Y., et al.,
2015. "Advances in Validation Methods for Remote Sensing Products of Land Surface Parameters." Journal of Remote Sensing 19 (1): 76-92.

[5] Liang, S. 2000. "Numerical Experiments on the Spatial Scaling Of Land Surface Albedo and Leaf Area Index." Remote Sensing Reviews 19 (1-4): 225-242.

[6] Liang, S., Fang, H., Chen, M., Shuey, C., Walthall, C., Daughtry, C., et al. 2002. "Validating MODIS Land Surface Reflectance and Albedo Products: Methods and Preliminary Results." Remote Sensing of Environment 83 (1): 149-162.

[7] Aman, A., Randriamanantena, H. P., Podaire, A., and Frouin, R. J. 1992. "Upscale Integration of Normalized Difference Vegetation Index: The Problem of Spatial Heterogeneity." IEEE Transactions on Geosciences and Remote Sensing 30 (2): 326-338.

[8] Bian, L., and Walsh, S. J. 1993. "Scale Dependencies of Vegetation and Topography in a Mountainous Environment of Montana." Professional Geographer 45 (1): 1-11.

[9] Friedl, M. A., Davis, F. W., Michaelsen, J., and Moritz, M. A. 1995. "Scaling and Uncertainty in the Relationship between the NDVI and Land Surface Biophysical Variables: An Analysis Using a Scene Simulation Model and Data from FIFE." Remote Sensing of Environment 54 (3): 233-246.

[10] VanDer Meer, F. D., Bakker, W. H., Scholte, K., Skidmore, A. K., De Jong, S. M., Clevers, J. G. P. W., et al. 2001. "Spatial Scale Variations in Vegetation Indices and Above-Ground Biomass Estimates: Implications for MERIS.” International Journal of Remote Sensing 22 (17): 3381-3396.

[11] Gu, Y. X., and Wylie, B. K. 2015. "Developing a 30-m Grassland Productivity Estimation Map for Central Nebraska Using 250-M MODIS and 30-M Landsat-8 Observations." Remote Sensing of Environment 171: 291-298.

[12] Hu, Z. L., and Islam, S. 1997. "A Framework for Analyzing and Designing Scale Invariant Remote Sensing Algorithms." IEEE Transactions on Geoscience and Remote Sensing 35 (3): 747-755.

[13] Zhang, X., Yan, G., Li, Q., Li, Z., Wan, H., and Guo, Z. 2006. "Evaluating the Fraction of Vegetation Cover Based 

Sensibility Analysis to Various Image Characteristics

on NDVI Spatial Scale Correction Model.” International Journal of Remote Sensing 27 (24): 5359-5372.

[14] Martonchik, J. V., Bruegge, C. J., and Strahler, A. H. 2000. "A Review of Reflectance Nomenclature Used in Remote Sensing.” Remote Sensing Reviews 19 (1-4): 9-20.

[15] Luan, H., Tian, Q., Yu, T., Hu, X., Huang, Y., Du, L., et al. 2013. "Modeling Continuous Scaling of NDVI Based on Fractal Theory." Spectroscopy and Spectral Analysis 33 (7): 1857-1862. (in Chinese).

[16] Luan, H., Tian, Q., Gu, X., Yu, T., and Hu, X. 2013. "Modeling Continuous Scaling of NDVI Based on Fractal Theory and GEOEYE-1 Image." Journal of Infrared and Millimeter Waves 32 (6): 538-544 + 549. (in Chinese).

[17] Zhang, R. H., Tian, J., Li, Z. L., Su, H. B., Chen, S. H., and Tang, X. Z. 2010. "Principles and Methods for the
Validation of Quantitative Remote Sensing Products." Science China Earth Sciences 53 (3): 741-751.

[18] Zhang, X. H. 2012. "Study on Spatial Heterogeneity and Scale Effect of Eucalyptus Forest Based on High Resolution Remote Sensing." Ph. D. thesis, Nanjing University, 49-50. (in Chinese).

[19] Chen, Y., and Chen, L. 2005. Fractal Geometry (2nd ed.). Beijing: Earthquake Press, 39-44 + 95-98. (in Chinese).

[20] Kim, G., and Barros, A. P. 2002. "Downscaling of Remotely Sensed Soil Moisture with a Modified Fractal Interpolation Method Using Contraction Mapping and Ancillary Data." Remote Sensing of Environment 83 (3): 400-413.

[21] Xie, H. P., and Sun, H. Q. 1997. "The Study on Bivariate Fractal Interpolation Functions and Creation of Fractal Interpolated Surfaces." Fractals 5 (4): 625-634. 